\title{
Challenges of Secondary Traumatic Stress in Mental Health Nurses Working in Kigali, Rwanda and Development of Management Strategies
}

\author{
J.D. Iyamuremye \\ Mental Health Division, Rwanda Biomedical Center, Ministry of Health, Rwanda
}

\section{Background}

A unique feature of mental health nurses' work involves exposure to clients' descriptions of and reactions to trauma, and these experiences may indirectly cause distress to the mental health worker. This phenomenon has been termed "secondary traumatic stress" (STS) (Perez, Jones, Englert \& Sachau, 2010). The effects of STS can be serious and permanent in mental health workers working with traumatized clients (Satkunanayagam, Tunariu \& Tribe, 2010).

\section{Description}

Two studies were conducted to assess STS in mental health workers in Rwanda. The first quantitative study explored STS in 180 mental health providers who cared for trauma survivors. This study was conducted in 2010 using a validated tool called the Trauma Attachment Belief Scale (TABS). TABS targets the respondents' current work environment and psychosocial support systems. The study involved improving self-awareness and connection by providing education on STS, as well as supervision and debriefing, thus improving work conditions and providing adequate communication and resources and support systems.

The second study added qualitative measures involving the description of mental health workers' experiences of STS. The study was guided by Constructive Self-Development Theory (Pearlman, 2003) and used an action research approach. The study focused on developing a sense of self-care and wellness using personal and professional strategies, which encouraged physical activities, relaxation techniques, meditation techniques, and guided visualization. The results of the study supported the development of an intervention model to support mental health workers in Rwanda in the management of secondary traumatic stress.

\section{Lessons Learned}

The results of this research exploring secondary traumatic stress supported the development, implementation and evaluation of an intervention model to manage STS in mental health workers in Rwanda.

\section{Conclusions}

Findings of these studies support the importance of protecting mental health workers from secondary traumatic stress when working with traumatized clients. The development and implementation of the two prevention strategies may attenuate secondary traumatic stress in Mental Health workers in Rwanda.

Key words: secondary traumatic stress (STS), management strategy, mental health workers, post traumatic stress, Rwanda 